\title{
Best constants in Lieb-Thirring inequalities: a numerical investigation
}

\author{
Antoine Levitt ${ }^{1}$
}

\begin{abstract}
We investigate numerically the optimal constants in Lieb-Thirring inequalities by studying the associated maximization problem. Using a monotonic fixed-point algorithm and a finite element discretization, we obtain radial trial potentials which provide lower bounds on the optimal constants. These results confirm existing conjectures, and provide insight into the behavior of the maximizers. Based on our numerical results, we formulate a complete conjecture about the best constants for all possible values of the parameters.
\end{abstract}

Mathematics Subject Classification (2010). Primary 35P15; Secondary 81Q10.

Keywords. Lieb-Thirring inequalities, finite elements.

\section{Introduction}

In this paper we study the family of Lieb-Thirring inequalities [20] and [21], which state that for any potential $V \in L^{\gamma+d / 2}\left(\mathbb{R}^{d}, \mathbb{R}\right)$ and non-negative exponent $\gamma$,

$$
\operatorname{tr}\left((-\Delta+V)_{-}^{\gamma}\right) \leq L_{\gamma, d} \int V_{-}^{\gamma+d / 2},
$$

where $V_{-}=\max (-V, 0)$ is the negative part of $V$, and $(-\Delta+V)_{-}^{\gamma}$ is the $\gamma$-th power of the negative part of the operator $(-\Delta+V)$, as defined by the functional calculus. In other words, $\operatorname{tr}\left((-\Delta+V)_{-}^{\gamma}\right)=\sum_{i}\left|\lambda_{i}\right|^{\gamma}$ is the $\gamma$-th moment of the negative eigenvalues of $-\Delta+V$.

This inequality was originally used by Lieb and Thirring in the case $\gamma=1, d=3$ as an important tool to prove the stability of fermionic matter in [20] (see [17] and [18] for an overview). The generalization (1) to any $\gamma$ and $d$ has since then attracted a great deal of attention (see for instance [11] for a review). Of particular interest are the values of $\gamma$ and $d$ for which this inequality holds, and the value of the optimal constants $L_{\gamma, d}$.

\footnotetext{
${ }^{1}$ Support from the grant ANR-10-BLAN-0101 of the French Ministry of Research is gratefully acknowledged.
} 
Despite the physical significance of the Lieb-Thirring inequality and the amount of mathematical research on the subject, a number of questions are still open. This paper aims to investigate some of these numerically. To our knowledge, this has not been done since the work of Barnes in the appendix of the original paper by Lieb and Thirring [21], in 1976.

It is easy to see that the bound (1) cannot hold for $\gamma<1 / 2$ when $d=1$ by scaling, and $\gamma=0$ when $d=2$ (because any arbitrarily small potential in two dimension creates at least one bound state). The inequality was proved for $\gamma>1 / 2$ in one dimension and $\gamma>0$ in other dimensions in the original paper by Lieb and Thirring [21]. The proof in the case $\gamma=1$ was recently greatly simplified by Rumin [25]. The case $\gamma=0, d \geq 3$ requires completely different methods and was proven independently by Cwikel [3], Lieb [15], and Rozenblum [24]. The limit case $\gamma=1 / 2, d=1$ remained unsolved for twenty years until it was finally settled by Weidl [27], and refined with a sharp constant by Hundertmark, Lieb and Thomas [9].

Inequality (1) can be interpreted as a comparison of the quantum mechanical energy of a system with Hamiltonian $-\Delta+V$ to its semiclassical approximation. The semiclassical regime is obtained by letting the Planck constant $\hbar$ tend to zero in the Hamiltonian $-\hbar \Delta+V$. In this paper, we have scaled $\hbar$ away from inequality (1) for convenience, and the corresponding limit is a large (or extended) $V$. In this limit, the eigenfunctions become localized in the region of the phase space defined by $p^{2}+V(x) \leq 0$ and explicit computations are possible. More precisely, using the Weyl asymptotics, one can prove

$$
\lim _{\mu \rightarrow \infty} \frac{\operatorname{tr}\left((-\Delta+\mu V)_{-}^{\gamma}\right)}{\int(\mu V)^{\gamma+d / 2}}=L_{\gamma, d, \mathrm{sc}}
$$

where

$$
L_{\gamma, d, \mathrm{sc}}=2^{-d} \pi^{-d / 2} \frac{\Gamma(\gamma+1)}{\Gamma(\gamma+d / 2+1)} .
$$

Therefore, denoting by $L_{\gamma, d}$ the optimal constant in (1), we have $L_{\gamma, d} \geq L_{\gamma, d \text {,sc. }}$. We set

$$
R_{\gamma, d}=\frac{L_{\gamma, d}}{L_{\gamma, d, \mathrm{sc}}} \geq 1
$$

The value of $R_{\gamma, d}$ describes by how much the quantum mechanical energy can exceed its semiclassical counterpart. In this paper, we investigate numerically the problem

$$
R_{\gamma, d}=\frac{1}{L_{\gamma, d, \mathrm{sc}}} \sup \left\{\operatorname{tr}\left((-\Delta+V)_{-}^{\gamma}\right), V \in L^{\gamma+d / 2}, \int V^{\gamma+d / 2}=1\right\}, \quad\left(P_{\gamma, d}\right)
$$

where we impose the condition

$$
\int V^{\gamma+d / 2}=1
$$


to eliminate the scaling invariance of the Lieb-Thirring inequality. As a global maximization of this non-concave functional is out of reach, we develop an algorithm to maximize it locally, and present numerical results for different starting points.

Several important facts about $R_{\gamma, d}$ are known. A scaling argument by Aizenman and Lieb [1] shows that $R_{\gamma, d}$ is decreasing with respect to $\gamma$. Laptev and Weidl [12] showed that $R_{\gamma, d}=1$ for $\gamma \geq 3 / 2$. Helffer and Robert [8] proved that $R_{\gamma, d}>1$ for $\gamma<1$ by expanding $\operatorname{tr}(-\Delta+V)_{-}^{\gamma}$ for the harmonic potential $V(x)=1-|x|^{2}$ in the semiclassical limit. From these results, we can deduce that for each dimension $d$ there exists a critical $\gamma_{c, d}$ such that

$$
\left\{\begin{array}{l}
R_{\gamma, d}>1 \quad \text { when } \gamma<\gamma_{c, d}, \\
R_{\gamma, d}=1 \quad \text { when } \gamma \geq \gamma_{c, d},
\end{array}\right.
$$

with

$$
1 \leq \gamma_{c, d} \leq \frac{3}{2}
$$

A trial potential of [21] provides a lower bound on $R_{\gamma, d}$ and therefore $\gamma_{c}$. In the appendix of this paper, Barnes solved numerically the restricted problem

$$
R_{\gamma, d, 1}=\frac{1}{L_{\gamma, d, \mathrm{sc}}} \sup _{V^{\gamma+d / 2}=1}\left|\lambda_{1}\right|^{\gamma},
$$

where $\lambda_{1}$ is the lowest eigenvalue of $-\Delta+V$. This is equivalent to restricting $\left(P_{\gamma, d}\right)$ to the potentials $V$ such that $-\Delta+V$ only has one negative eigenvalue. The solution $V_{\gamma, d, 1}$ of this problem is negative, radial and only has bound state, i.e. $-\Delta+V_{\gamma, d, 1}$ only has one negative eigenvalue. The corresponding $R_{\gamma, d, 1}$ is decreasing with $\gamma$, and intersects 1 at a critical $\gamma_{c, d, 1}$. In low dimensions, $\gamma_{c, 1,1}=\frac{3}{2}, \gamma_{c, 2,1} \approx 1.165$, $\gamma_{c, 3,1} \approx 0.863$ (our numerical results agree with these values). Therefore, $\gamma_{c, 1}=\frac{3}{2}$ and $\gamma_{c, 2} \geq 1.165$, but nothing can be said about $\gamma_{c, d}$ for $d \geq 3$. A famous conjecture of Lieb and Thirring states that $R_{1,3}=1$, i.e. $\gamma_{c, 3}=1$. This would imply improved bounds on the energy of a system of fermions, and is of great importance to the Thomas-Fermi theory [16].

Better upper bounds on $R_{\gamma, d}$ have also been derived recently. For instance, it is proven in [6] that $R_{\gamma, d} \leq \frac{2 \pi}{\sqrt{3}} \approx 3.63$ for $\gamma \geq \frac{1}{2}, R_{\gamma, d} \leq \frac{\pi}{\sqrt{3}} \approx 1.82$ for $\gamma \geq 1$.

Despite these advances on upper bounds, not much is known about lower bounds for $R_{\gamma, d}$, except for the one-bound state potential $V_{\gamma, d, 1}$ of [21] and the asymptotic result in the semiclassical limit of [8]. In this paper, we attempt to bridge the gap between these two results by looking numerically for maximizers of the variational problem $\left(P_{\gamma, d}\right)$. To our knowledge, this is the first numerical study of the LiebThirring inequalities since the work of Barnes [21] (and unpublished recent work by Arnold and Dolbeault [4] in 1D). 
The method we use is a finite element discretization of a natural fixed point algorithm. We describe this algorithm, and specialize it to the case of radial potentials. Then we discretize it, and use it to obtain numerical results. The critical points we obtain are the natural generalization of the potential with one bound state obtained by Barnes. They serve as a partial bridge between this potential and asymptotic results, and yield new lower bounds on $R_{\gamma, d}$.

Acknowledgments. I would like to thank Mathieu Lewin and Éric Séré for their help and advice.

\section{The optimization algorithm}

2.1. Optimization scheme. Let us denote

$$
E(V)=\operatorname{tr}\left((-\Delta+V)_{-}^{\gamma}\right)
$$

so that

$$
L_{\gamma, d}=\sup \left\{E(V), V \in L^{\gamma+d / 2}, \int V^{\gamma+d / 2}=1\right\} .
$$

Let $S_{p}$ be the set of symmetric operators on $L^{2}\left(\mathbb{R}^{d}\right)$ with finite Schatten norm

$$
\|\left.\tau\right|_{p}=\operatorname{tr}\left(|\tau|^{p}\right)^{1 / p}
$$

The fixed point algorithm we use is based on the following property.

Proposition 1. For any $V \in L^{\gamma+d / 2}\left(\mathbb{R}^{d}, \mathbb{R}\right)$ and any $\gamma \geq 1$,

$$
E(V)^{1 / \gamma}=\max \left\{-\operatorname{tr}((-\Delta+V) \tau) / \tau \in S_{\gamma^{\prime}}, \tau \geq 0,\|\tau\|_{\gamma^{\prime}}=1\right\},
$$

where

$$
\gamma^{\prime}=\frac{\gamma}{\gamma-1}
$$

is the Hölder conjugate of $\gamma$.

Proof. For any $V \in L^{\gamma+d / 2}, \tau \in S_{\gamma}$ with $\tau \geq 0,\|\tau\|_{\gamma^{\prime}}=1$,

$$
\begin{aligned}
-\operatorname{tr}((-\Delta+V) \tau) & \leq \operatorname{tr}\left((-\Delta+V)_{-} \tau\right) \\
& \leq\left\|(-\Delta+V)_{-}\right\|_{\gamma}\left\|_{\tau}\right\|_{\gamma^{\prime}} \\
& =\operatorname{tr}\left((-\Delta+V)_{-}^{\gamma}\right)^{1 / \gamma} \\
& =E(V)^{1 / \gamma}
\end{aligned}
$$

and the equality is achieved when

$$
\tau=K_{\tau}(-\Delta+V)_{-}^{\gamma-1}
$$

where $K_{\tau}$ is a normalization constant chosen to ensure that $\|\tau\|_{\gamma^{\prime}}=1$. 
From this, we see that, when $\gamma \geq 1$,

$$
\begin{array}{r}
L_{\gamma, d}^{1 / \gamma}=\sup \left\{-\operatorname{tr}((-\Delta+V) \tau) / \tau \in S_{\gamma^{\prime}},\|\tau\|_{\gamma^{\prime}}=1, \tau \geq 0,\right. \\
\left.V \in L^{\gamma+d / 2}, \int V^{\gamma+d / 2}=1, V \leq 0\right\} .
\end{array}
$$

What we gain from this formulation is that we can explicitly maximize

$$
-\operatorname{tr}((-\Delta+V) \tau)
$$

with respect to $V$ and $\tau$ separately. Indeed, for a fixed $V$, the maximum with respect to $\tau$ is given by (3), and for a fixed $\tau$, the maximum with respect to $V$ is given by

$$
V(x)=-K_{V} \tau(x, x)^{\frac{1}{\gamma+d / 2-1}},
$$

where $K_{V}$ is a normalization parameter chosen to ensure that

$$
\int V^{\gamma+d / 2}=1
$$

and $\tau(x, y)$ is the integral kernel of $\tau$.

This suggests the following maximization scheme. Given an approximate maximum $V_{n}$, we set

$$
\tau_{n}=K_{\tau}\left(-\Delta+V_{n}\right)_{-}^{\gamma-1}
$$

and

$$
V_{n+1}(x)=-K_{V} \tau_{n}(x, x)^{\frac{1}{\gamma+d / 2-1}},
$$

and iterate. Explicitly, this is described by the following algorithm.

Algorithm 1 (Maximization algorithm).

(1) Compute the negative eigenvalues $\lambda_{i}$ and thu corresponding eigenvectors $\psi_{i}$ of $-\Delta+V_{n}$

(2) Set

$$
\rho_{n}=\sum_{i}\left(-\lambda_{i}\right)^{\gamma-1} \psi_{i}^{2}
$$

(3) Compute

$$
K_{n}=\left\|\rho_{n}^{\frac{1}{\gamma+d / 2-1}}\right\|_{\gamma+d / 2}^{-1}=\left(\int \rho_{n}^{\frac{\gamma+d / 2}{\gamma+d / 2-1}}\right)^{-\frac{1}{\gamma+d / 2}}
$$

(4) Set $V_{n+1}=-K_{n} \rho_{n}^{\frac{1}{\gamma+d / 2-1}}$. 
By construction, this algorithm ensures that

$$
\begin{aligned}
E\left(V_{n+1}\right)^{1 / \gamma} & =-\operatorname{tr}\left(\left(-\Delta+V_{n+1}\right) \tau_{n+1}\right) \\
& \geq-\operatorname{tr}\left(\left(-\Delta+V_{n}\right) \tau_{n+1}\right) \\
& \geq-\operatorname{tr}\left(\left(-\Delta+V_{n}\right) \tau_{n}\right) \\
& =E\left(V_{n}\right)^{1 / \gamma} .
\end{aligned}
$$

Therefore, the sequence $E\left(V_{n}\right)$ is non-decreasing, and since it is bounded from above, it converges. In general though, $V_{n}$ may not converge. We give examples in Section 4 where, because of the translation invariance of the functional, $V_{n}$ splits into two bumps that separate from each other. Even when this behavior is forbidden, for instance by imposing a finite domain as we do in numerical computations, a rigorous convergence analysis of the algorithm is still an open problem.

Note that, even if Algorithm 1 was derived from Proposition 1 for $\gamma \geq 1$, it remains a reasonable algorithm when $\gamma<1$. In this case, though, the monotonicity of $E\left(V_{n}\right)$ is not guaranteed.

This algorithm can be seen as a fixed-point scheme for the critical points of the maximization problem. Indeed, the Euler-Lagrange equations for $\left(P_{\gamma, d}\right)$ are

$$
V(x)=-K_{V}\left[(-\Delta+V)_{-}^{\gamma-1}(x, x)\right]^{\frac{1}{\gamma+d / 2-1}} \text {. }
$$

This is a self-consistent set of equations similar to systems such as the HartreeFock equations of quantum chemistry [19]. Our algorithm is similar in spirit to the Roothaan method [23]. In our case, at least for $\gamma \geq 1$, the scheme monotonically increases the objective function. Therefore, the oscillatory behavior often seen in the Hartree-Fock model [2] and [14] cannot occur here. Even for $\gamma<1$, we did not see any such oscillations numerically, and always observed linear convergence (i.e. $\left\|V_{n}-V_{\infty}\right\| \leq v^{n}$ for some $v, 0<v<1$ ), or slow separation of bumps, as will be discussed in Section 4.

We also note that this algorithm is used in the context of the Lieb-Thirring inequalities by Arnold and Dolbeault in 1D; see [4].

2.2. Radial algorithm. Most of our multidimensional computations were done in a radial setting. To see why this is possible, consider the above iteration for $d \geq 2$, when $V_{n}$ is radial. Then, the Laplacian splits into $\Delta_{r}+\frac{1}{r^{d-1}} \Delta_{\theta}$, where $\Delta_{r}$ is the radial Laplacian, and $\Delta_{\theta}$ the Laplace-Beltrami operator on $S^{d-1}$. The eigenvectors of $\Delta_{\theta}$ are explicitly known to be the spherical harmonics, labeled by the integers $\ell$ and $m$. Since $V_{n}$ is radial, $-\Delta_{r}+V_{n}$ commutes with $\Delta_{\theta}$ and can therefore be diagonalized in the same basis (separation of variables). We write these eigenvectors in the form

$$
\psi_{i, \ell, m}(x)=\varphi_{i, \ell}(|x|) J_{\ell, m}\left(\frac{x}{|x|}\right)
$$


where $J_{\ell, m}$ is the spherical harmonic of degree $\ell$ and order $m$. The radial parts $\varphi_{i, \ell}$ satisfy the equation

$$
-\frac{1}{r^{d-1}}\left(r^{d-1} \varphi_{i, \ell}^{\prime}\right)^{\prime}+\frac{\ell(\ell+d-2)}{r^{2}} \varphi_{i, \ell}+V \varphi_{i, \ell}=\lambda_{i, \ell} \varphi_{i, \ell},
$$

and each $\lambda_{i, \ell}$ has multiplicity

$$
h(d, \ell)=\left(\begin{array}{c}
d+\ell-1 \\
\ell
\end{array}\right)-\left(\begin{array}{c}
d+\ell-3 \\
\ell-2
\end{array}\right)
$$

see [26].

Therefore, we can obtain all the negative eigenvectors and eigenvalues by solving only (5). Furthermore, since the lowest eigenvalue decreases as $\ell$ increases, we can iterate over $\ell$ and stop whenever the lowest eigenvalue becomes positive. Next, we compute

$$
\begin{aligned}
\rho_{n}(x) & =\sum_{\ell} \sum_{i} \sum_{m}\left(-\lambda_{i, \ell}\right)^{\gamma-1} \psi_{i, \ell, m}(x)^{2} \\
& =\sum_{\ell} h(d, \ell) \sum_{i}\left(-\lambda_{i, \ell}\right)^{\gamma-1} \varphi_{i, \ell}(r)^{2}
\end{aligned}
$$

where the sum only ranges over all negative eigenvalues. This is again radial, and therefore so is $V_{n+1}$. To summarize, we have the following algorithm.

Algorithm 2 (Radial maximization algorithm).

(1) Compute all the negative eigenvalues $\lambda_{i, \ell}$ and associated eigenvectors $\varphi_{i, \ell}$ of (5) by increasing $\ell$ until the lowest eigenvalue $\lambda_{0, \ell}$ becomes positive.

(2) Set

$$
\rho_{n}=\sum_{\ell} \sum_{i} h(d, \ell)\left(-\lambda_{i, \ell}\right)^{\gamma-1} \varphi_{i, \ell}^{2}
$$

(3) Compute

$$
K_{n}=\left\|\rho_{n}^{\frac{1}{\gamma+d / 2-1}}\right\|_{\gamma+d / 2}^{-1}=\left(\left|\$_{d-1}\right| \int \rho_{n}^{\frac{\gamma+d / 2}{\gamma+d / 2-1}}(r) \mathrm{d} r\right)^{-\frac{1}{\gamma+d / 2}} .
$$

(4) Set $V_{n+1}=-K_{N} \rho_{n}^{\frac{1}{\nu+d / 2-1}}$.

Note that this algorithm is not an approximation: the iterates generated by this algorithm coincide with the ones from Algorithm 1 when the initial guess $V_{0}$ is radial. 


\section{Discretization}

3.1. Galerkin basis and weak formulation. To discretize this algorithm, we use a Galerkin finite element basis on which we expand $V$ and the eigenvectors. This variational discretization has the advantage that numerical computations can provide accurate lower bounds on the best constants $R_{\gamma, d}$. A disadvantage is that the algorithm we use involves taking powers of functions. There is no exact way to do that in a Galerkin basis, and we must use an approximation, which causes a loss of accuracy in the fixed-point algorithm.

For the non-radial 1D and 2D cases, we simply use standard finite elements. The radial case is less standard, and we must derive a weak formulation of (5). There are several possibilities here. The simplest is to use a change of variable

$$
\varphi(r)=r^{\frac{d-1}{2}} \varphi(r)
$$

to transform the equation back to the more standard form

$$
-\varphi^{\prime \prime}+\frac{\left(l+\frac{d-1}{2}\right)\left(l+\frac{d-3}{2}\right)}{r^{2}} \varphi+V(r) \varphi=\lambda \varphi .
$$

We obtain a weak formulation by multiplying by a test function $u$ and integrating:

$$
\int\left[\varphi^{\prime} u^{\prime}+\left(\frac{\left(l+\frac{d-1}{2}\right)\left(l+\frac{d-3}{2}\right)}{r^{2}}+V\right) \varphi u\right]=\lambda \int \varphi u .
$$

Expanding the function $\varphi$ on a Galerkin basis $\varphi(r)=\sum_{i} x_{i} \chi_{i}(r)$, this problem transforms into the generalized matrix eigenvalue problem

$$
A x=\lambda M x,
$$

where

$$
A_{i j}=\int \chi_{i}^{\prime} \chi_{j}^{\prime}+\left(\frac{\left(l-\frac{d-1}{2}\right)\left(l+\frac{d-3}{2}\right)}{r^{2}}+V\right) \chi_{i} \chi_{j}
$$

and

$$
M_{i j}=\int \chi_{i} \chi_{j}
$$

are the stiffness and mass matrices. 
When $V$ is expanded on the same basis, we can compute the matrix elements, solve the eigenvalue problem (7), and transform back to $\varphi$. However, for $d=2, l=0$, $\varphi$ behaves like $\sqrt{r}$ at 0 , and the singularity of the derivative prevents an accurate discretization.

Another possibility is to obtain a weak formulation of (5) directly. We have to remember that since the $\varphi$ functions are only radial parts of a $d$-dimensional function, the $L^{2}$ inner product between two functions $\varphi_{1}$ and $\varphi_{2}$ is

$$
\int \varphi_{1} \varphi_{2} r^{d-1}
$$

This has to be taken into account in the weak formulation in order to obtain a selfadjoint equation. Multiplying (5) by $r^{d-1} u$, where $u$ is a test function, and integrating by parts, we obtain

$$
\int\left[r^{d-1} \varphi^{\prime} u^{\prime}+\left(\frac{l(l+d-2)}{r^{2}}+V\right) r^{d-1} \varphi u\right]=\lambda \int r^{d-1} \varphi u .
$$

Then, as with (6), we can transform this into a matrix equation and solve it. The disadvantage is that the integrations required for the assembly step are more involved, and therefore we only use it for the case $d=2, l=0$ where the other method fails.

3.2. Finite elements. We use a Finite Element basis of piecewise linear functions on a grid of $[0, L]$. The grid we use is a nonuniform grid of $N$ points, with more points around 0 , to accommodate for the singularity of (5). $L$ is chosen large enough so that all the eigenvectors associated to negative eigenvalues of $-\Delta+V$ can be represented accurately in the basis. But if $\psi$ is an eigenvector with negative eigenvalue $\lambda$, then, $\psi$ decays as $\exp (-\sqrt{-\lambda} r)$. This shows that the discretization is problematic for eigenvalues close to zero, as we shall see in Section 4.

For our piecewise linear basis functions, it is easy to compute the matrix elements (8) and (9) when $V_{n}$ is expanded on this same basis $\chi_{i}$. We then solve the eigenproblem (7), and obtain the eigenvectors. To expand $\rho^{\frac{1}{\gamma+d / 2-1}}$ on the basis, we simply chose the expansion that is exact on the grid points. Then the normalization can be performed exactly, and the iteration is carried out.

We present convergence results in Figure 1. As expected from piecewise linear basis functions, we obtain $O(1 / N)$ convergence of the eigenfunctions $\varphi$ in $H^{1}$ norm, and $O\left(1 / N^{2}\right)$ convergence of the eigenvalues $\lambda$ (and therefore of $E(V)$ ). For a given stepsize, the discretization error is exponential with respect to $L$, with a decay rate equal to the decay rate of the eigenfunctions, i.e. $\sqrt{-\lambda}$. Although we used a uniform grid in one dimension in Figure 1, similar results were checked to hold for our non-uniform grid in $d$ dimensions, using the weak formulation (6), except for $d=2, l=0$, where the convergence was slower and the weak formulation (10) had to be used to get the same convergence rates. 

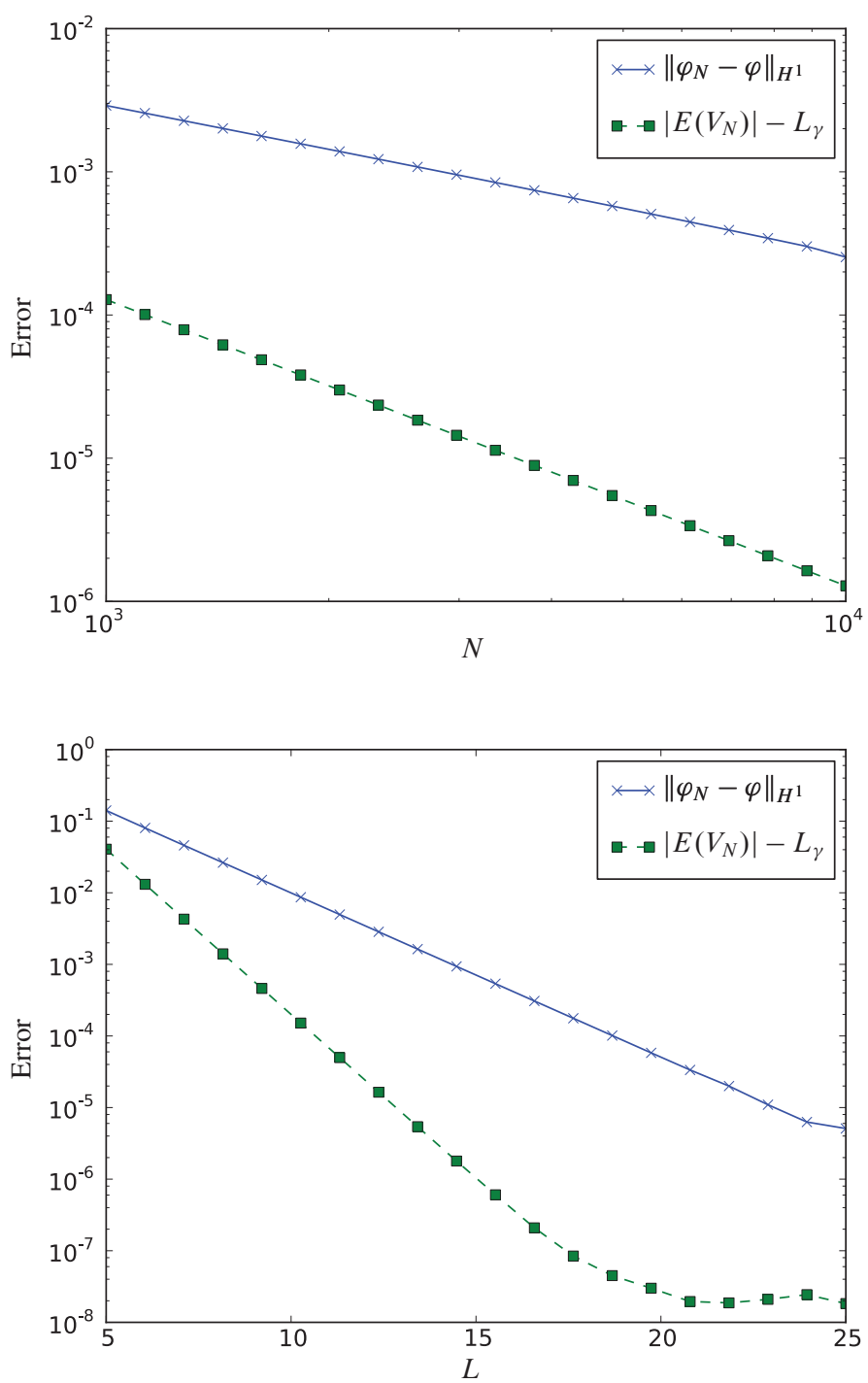

Figure 1. Convergence analysis with respect to $N$ and $L$. The figure on the left illustrates the convergence of the eigenfunctions and eigenvalues with respect to the number of grid points $N$, with a fixed $L=40$, large enough to cause a negligible error. The slopes, found by linear regression, are -1.043 for the eigenvectors and -1.999 for the eigenvalues, close to their theoretical values of -1 and -2 . The figure on the right illustrates the convergence of the eigenfunctions and eigenvalues with respect to the domain size, with constant stepsize $h=5 \times 10^{-4}$. The slope for the eigenvectors is $-0.5285 \approx \sqrt{-\lambda}=-0.5283$. When the domain size gets large enough, the error due to $h$ dominates, and causes a plateau. 
3.3. Diagonalization. The most computationally challenging step in our algorithm is the problem of computing all the negative eigenvalues and associated eigenvectors of a generalized eigenvalue problem $A x=M x$, where $A$ and $M$ are large sparse symmetric matrices. The spectrum of $A$ consists of $n$ negative eigenvalues, and a large number of positive eigenvalues, which can be seen as perturbations of the spectrum of the discrete Laplacian.

In order to compute the $n$ negative eigenvalues, where $n$ is unknown, we compute the $k$ lowest eigenvalues, check if the $k$-th one is positive, and repeat the process with a larger $k$ if not. The computation of the $k$ first eigenvalues can be done by standard packages such as ARPACK; see [13]. However, standard algorithms such as Arnoldi iteration are not suited for the computation of inner eigenvalues, and one has to solve for the largest eigenvalues of the shifted and inverted problem $(A-\sigma)^{-1} x=$ $(\lambda-\sigma)^{-1} x$ instead to ensure reasonably fast convergence. This requires an adequate shift (one that is close to the bottom of the spectrum of $A$ ). Even with this shiftand-invert strategy, the group of eigenvalues one needs to locate is not well-separated from the rest of the spectrum, and becomes less and less so as $L$ increases. This leads to slow convergence for large $L$.

3.4. Implementation. Using the Numpy/Scipy libraries [10], we implemented the algorithm in Python, with the ARPACK eigenvalue solver [13].

3.5. Error control. Our numerical methods introduce errors. For each $V$ we construct, we can get a lower bound of $L_{\gamma, d}$, provided we can accurately compute $E(V)$ and the normalization constant $\int V^{\gamma+d / 2}$.

Because the basis functions we use are piecewise linear, all the integrals involved in our computations, such as $\int V^{\gamma+d / 2}$, can be reduced to integrals of piecewise polynomials, which can be computed explicitly, up to machine precision.

It now remains to examine the accuracy of $E(V)$, i.e. of the computation of eigenvalues of $-\Delta+V$. Because we use a Galerkin discretization, the min-max theorem guarantees that the eigenvalues of the matrix problem we solve are larger than the true eigenvalues. The matrix problem is solved using ARPACK, which yields a collection of orthogonal approximate eigenvectors. Again using the minmax theorem, the eigenvalues of the submatrix formed by restricting the eigenvalue problem to the subspace spanned by the approximate eigenvectors are upper bounds on the true eigenvalues of $-\Delta+V$. This submatrix is of modest size, and its eigenvalues can be computed accurately by ARPACK.

For the reasons mentioned above, we believe our method to yield lower bounds on $L_{\gamma, d}$ with an accuracy comparable to machine precision (about $10^{-16}$ ). However, guaranteed lower bounds would require methods such as interval arithmetic, which we have not implemented. 


\section{Numerical results}

We used the algorithm described above to compute critical points of the variational problem $\left(P_{\gamma, d}\right)$, that is, a solution to the self-consistent equation (4). Our strategy was to find a critical point by running the algorithm on a suitable initial potential $V_{0}$ (for instance, a Gaussian of specified width). Once convergence is achieved for a specific $\gamma$, we can run the algorithm for $\gamma+\Delta \gamma$, using as initial potential the one found for $\gamma$. We have been unable to analytically prove the correctness of this method, for instance by checking the conditions of the implicit function theorem. However, we found it reliable enough for our purpose, as long as $\Delta \gamma$ was chosen small enough.

4.1. The 1D case. In one dimension we simply used a grid of size $[-L, L]$ with Dirichlet boundary conditions. We reproduced the potential with one bound state $V_{\gamma, 1,1}$ of [21] by using for $V_{0}$ a Gaussian of relatively small variance (see Figure 2). In this case, the algorithm converges linearly (i.e. $\left\|V_{n+1}-V_{n}\right\| \approx v^{n}$, for some convergence rate $v<1$ ), and very quickly (about twenty iterations to achieve machine precision).

Contrary to what we observe in higher dimensions, initializing the algorithm with a Gaussian of larger variance did not make the algorithm converge to other critical points. Instead, it leads to a slow divergence where "bumps" separate, each bump corresponding to the potential of $V_{\gamma, 1,1}$ (see Figure 3).

This effect occurred regardless of the value of $\gamma$ in the range $\gamma \in(1 / 2,3 / 2)$. The divergence appears to be logarithmic. More precisely, a numerical fit showed the asymptotic relationship for the distance $L_{n}$ between the two bumps

$$
L_{n} \approx \frac{1}{2 \sqrt{-\lambda}} \log n
$$

where $\lambda$ is the unique negative eigenvalue of $-\Delta+V_{\gamma, 1,1}$.

This strikingly simple relationship can heuristically be understood by the fact that, because the eigenfunctions $\varphi_{1}$ and $\varphi_{2}$ corresponding to the two bumps have exponential decay with decay constant $\sqrt{-\lambda}$, their interaction is of order $\exp \left(-\sqrt{-\lambda} L_{n}\right)$. Due to cancellations, this interaction leads to a correction of order $\exp \left(-2 \sqrt{-\lambda} L_{n}\right)$ in $V_{n+1}$, and we have the approximate relationship $L_{n+1} \approx L_{n}+K \exp \left(-2 \sqrt{-\lambda} L_{n}\right)$, which yields (11). A rigorous explanation of this is an interesting question.

Based on these results, we conjecture that $V_{\gamma, d, 1}$ is, up to translation, the only maximizer of the functional. This would imply that

$$
R_{\gamma, 1}=2\left(\frac{\gamma-1 / 2}{\gamma+1 / 2}\right)^{\gamma-1 / 2}
$$

for $\gamma \leq \frac{3}{2}$, in agreement with the original conjecture of Lieb and Thirring [21]. 



Figure 2. Linear convergence from a Gaussian initial data towards $V_{\gamma, d, 1}$. This plot is for $\gamma=1.2$. 

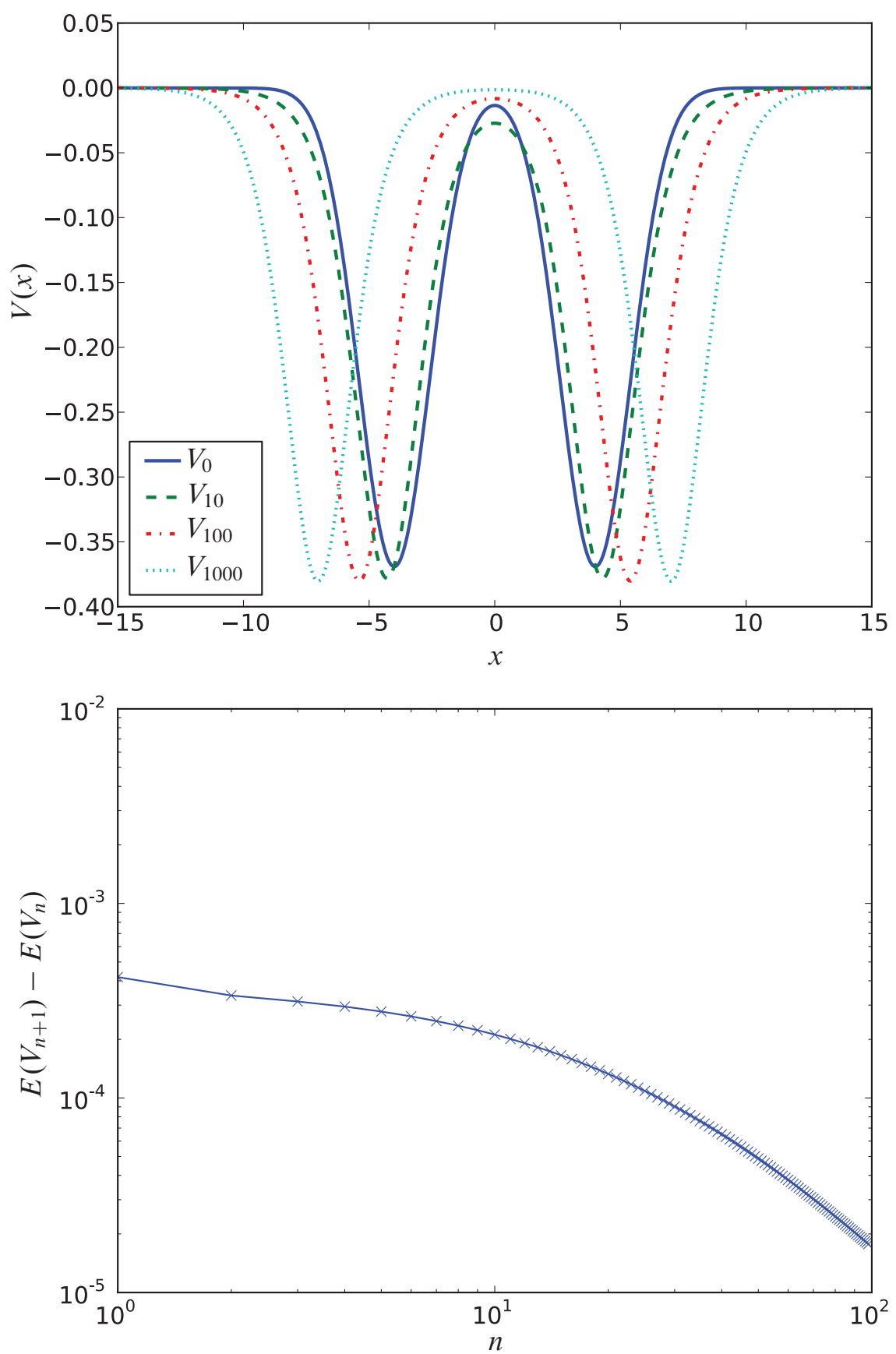

Figure 3. Divergence from a sum of Gaussian bumps at $\gamma=1.2$. Initializing to a single Gaussian of large width yields similar results. The two bumps separate slowly, until the finiteness of $L$ forces convergence. Although not displayed here, the asymptotic logarithmic divergence (11) can be checked graphically, e.g. the spacing between $V_{100}$ and $V_{1000}$ is the same than between $V_{1000}$ and $V_{10000}$. The asymptotic slope of the log-log convergence plot is -1 , which fits with the heuristic arguments given. 
4.2. The 2D case. Due to the high cost of accurately solving the maximization problem in more than one dimension, we only obtained partial results in the nonradial $2 \mathrm{D}$ case. The results indicate that the algorithm either converges to radial critical points, or form slowly separating bumps, as in one dimension. We have been unable to find any example of non-radial critical points, although this does not mean that they do not exist. An example of separation in bumps is provided in Figure 4.

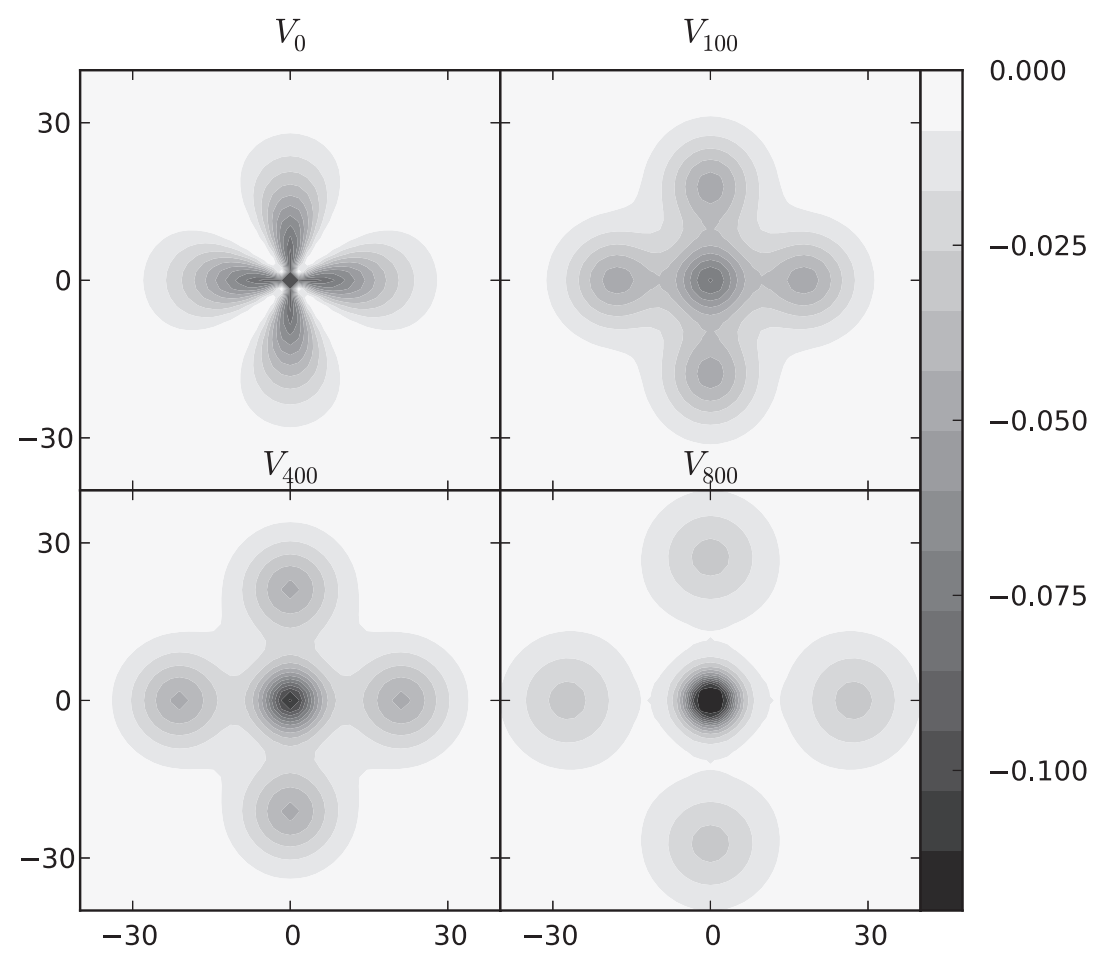

Figure 4. Separation in bumps of 2D non-radial initial data at $\gamma=1.2$, computed from Algorithm 1 with standard FEM. The initial data is taken to be a Gaussian in $r$ multiplied by an angular factor $(1+\cos (4 \theta))$.

In the radial case, we followed branches of critical points of $\left(P_{\gamma, d}\right)$ using the continuation method on $\gamma$ we described in Section 4. We found this branch continuation procedure robust. By varying the shape of the initial data, and in particular its width, we were able to obtain different branches of critical points. Generally speaking, as the width of the initial potential increases, the number of negative eigenvalues of $-\Delta+V$ increases.

We display the energy of these branches as a function of $\gamma$ in Figure 5.

First, we see that as the number of eigenvalues increases, $R\left(V_{\gamma}\right)$ tends to 1 , the semiclassical limit. For a given $\gamma$, the Lieb-Thirring constant will be given by the supremum of $R\left(V_{\gamma}\right)$ over such curves. From the branches depicted here, we see 
that the supremum is always given by either $V_{\gamma, d, 1}$ for $\gamma<\gamma_{c}^{1} \approx 1.16$, and by the semiclassical limit for $\gamma>\gamma_{c}^{1}$. All the other curves are below these two (although not depicted in this zoomed plot, this holds true for $0.5<\gamma<1.5$ ).

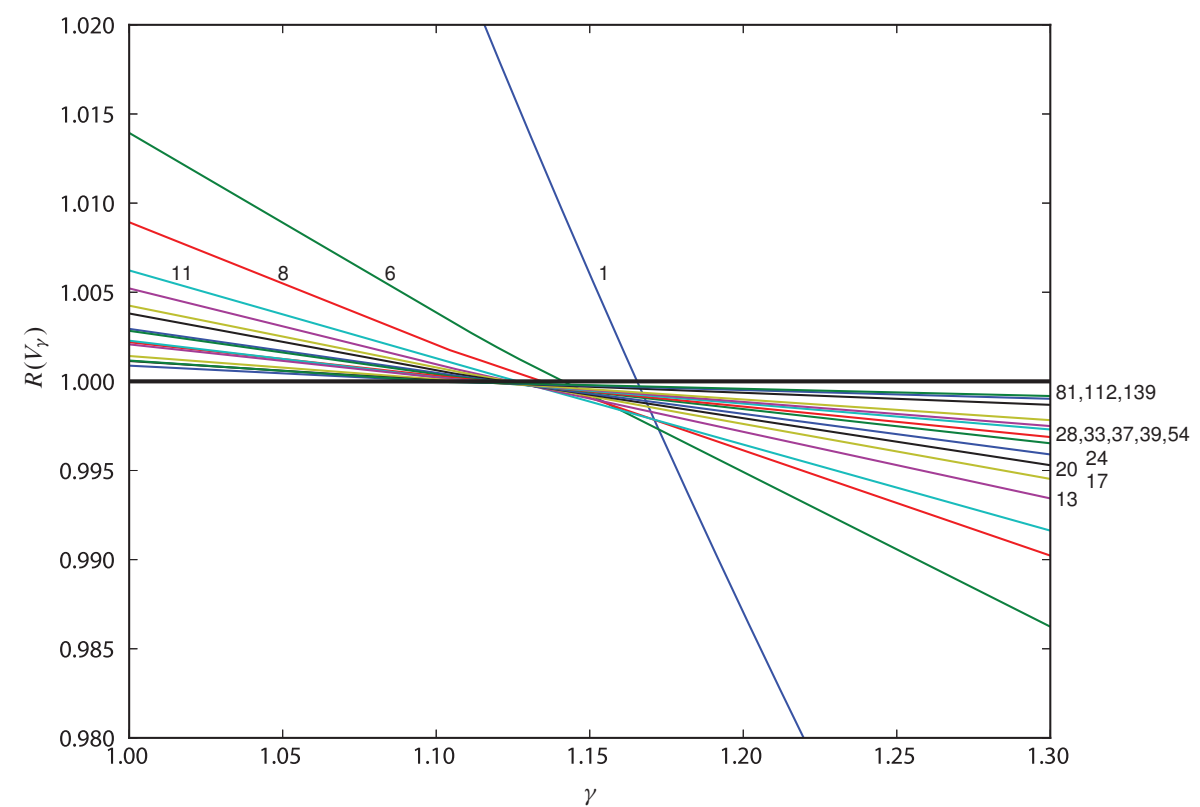

Figure 5. $R(V)$ as a function of $\gamma$ for $d=2$ using the branch continuation procedure in the radial setting (Algorithm 2). The branches are labeled by the number of bound states they have. Based on these results, the maximizer seems to be $V_{\gamma, d, 1}$, up to $\gamma \approx 1.16$. Above this, no branches were above the semiclassical regime $R=1$.

Table 1. Values $\gamma_{c, 2, k}$ of $\gamma$ at which some branches with $k$ bound states cross the threshold $R=1$.

\begin{tabular}{c|c|c|c|c|c|c|c|}
\hline$k$ & 1 & 4 & 6 & 8 & 11 \\
\hline \multicolumn{1}{c|}{$\gamma_{c, 2, k}$} & 1.165 & 1.150 & 1.141 & 1.135 & 1.126 \\
\hline$k$ & 13 & 17 & 28 & 54 & 81 & 139 \\
\hline$\gamma_{c, 2, k}$ & 1.124 & 1.124 & 1.119 & 1.110 & 1.105 & 1.100 \\
\hline
\end{tabular}

It is important to keep in mind that these branches only represent critical points of the functional. They are generically local maxima with respect to radial perturbations, but might not be stable with respect to non-radial ones. For instance, Figure 6 depicts what happens when the radial potential with eight bound states is perturbed nonradially by a Gaussian multiplicative noise, with $\gamma=1.1$. Because the potential with one bound state has a higher energy, the potential splits into eight bumps. Performing the same experiment $\gamma$ larger than about 1.17, where the potential with one bound 
state has a lower energy, when the potential with eight bound states has a larger energy, we found that no splitting occurs, which suggests that the potentials are stable beyond their crossing with the one with one bound state.

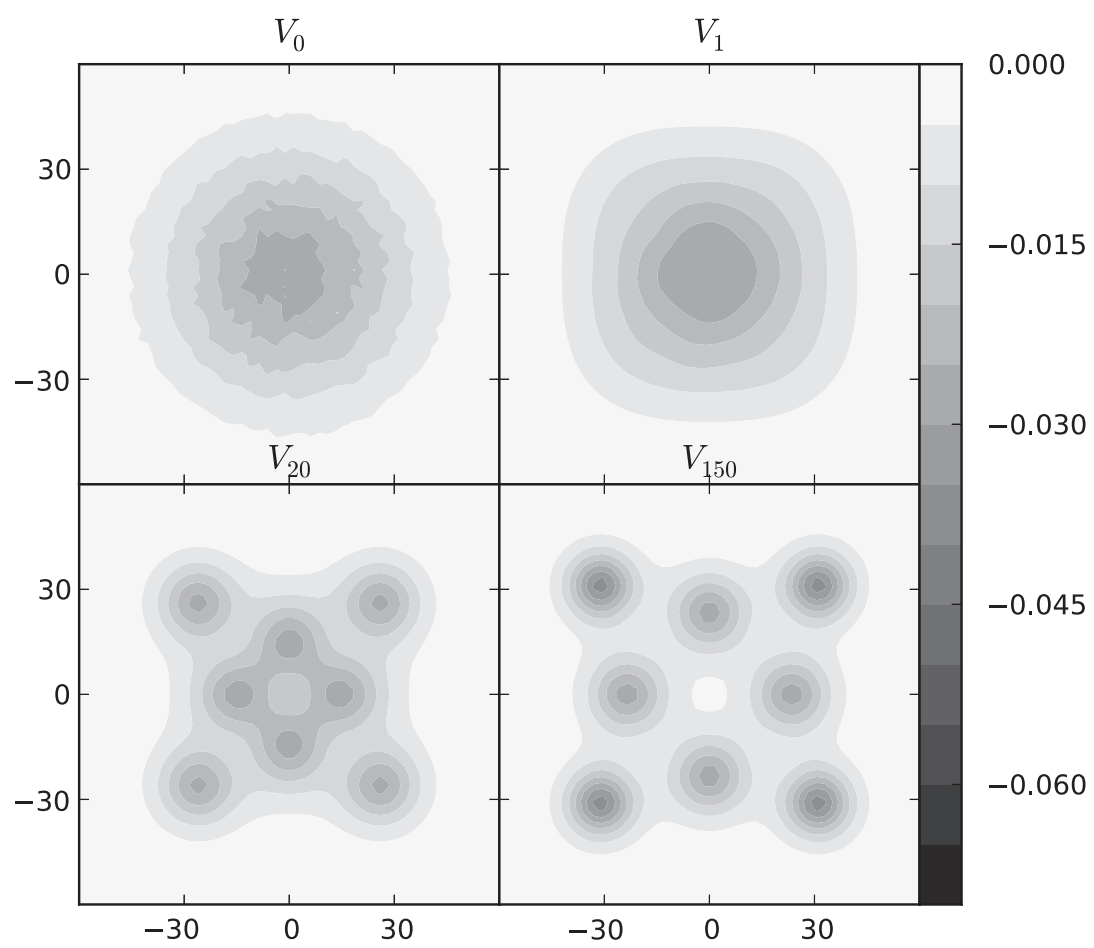

Figure 6. Separation in bumps of the randomly perturbed radial potential with eight bound states, $\gamma=1.1$.

Based on our numerical results, we conjecture that $\gamma_{c}=\gamma_{c}^{1} \approx 1.16$. The only way this conjecture could be false is if some other curve is above the one with one bound state. We have not been able to find such a curve.

4.3. The 3D case. Due to the high cost of computation, we were unable to get meaningful results in the non-radial setting, and only present our findings in the radial case. Some of the radial potentials we found are presented in Figure 7, with numerical data about the crossings of the curves in Table 2.

Contrary to the dimension 2, here some potentials with a higher number of bound states have a higher $R$ than $V_{\gamma, d, 1}$. The corresponding curves in the $\gamma-R$ plane are flatter and flatter, and intersect at a sequence of increasing $\gamma_{c}^{k}$. This sequence seems to accumulate at 1 , in accordance to Helffer and Robert's result [8], which predicts the existence of similar potentials with a $R$ larger than 1 for every $\gamma<1$ in the nearly semiclassical regime. However, their study of an harmonic potential of varying 
width showed a highly oscillatory behavior of $E(V)$ with respect to the width of the potential. Although our numerical methods do not permit us to investigate this regime (it would require a very large domain size, with a correspondingly large number of points, and a very poor conditioning of the eigenvalue problem), we expect the same behavior to occur. This means that the computation of the maximizing branches (here, those with 1, 5, 14 and 140 bound states) becomes harder and harder.

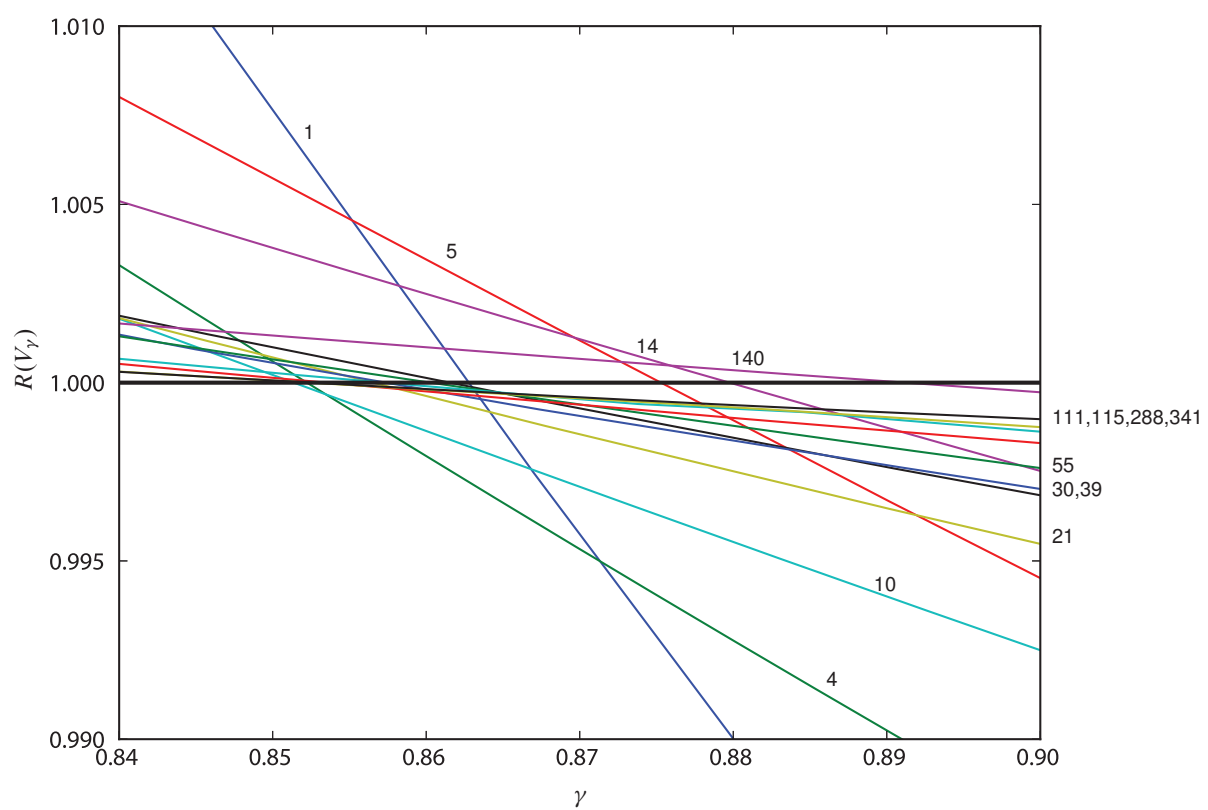

Figure 7. $R(V)$ as a function of $\gamma$ for $d=3$, with the same methodology as in Figure 5 . As $\gamma$ increases, different branches become maximizers, until $\gamma=1$. The fact that only some branches are above the threshold $R=1$ when the number of bound states increases results from the highly oscillatory behavior near $\gamma=1$, as predicted in [8].

Table 2. Values $\gamma_{c, 3, k}$ of $\gamma$ at which some branches with $k$ bound states cross the threshold $R=1$.

\begin{tabular}{|c|c|c|c|c|c|c|c|}
\hline$k$ & 1 & 4 & 5 & 10 & 14 \\
\hline$\gamma_{c, 3, k}$ & 0.863 & 0.852 & 0.875 & 0.851 & 0.880 \\
\hline$k$ & 21 & 30 & 55 & 111 & 140 & 341 \\
\hline$\gamma_{c, 3, k}$ & 0.857 & 0.862 & 0.860 & 0.854 & 0.891 & 0.853 \\
\hline
\end{tabular}

The relative energies of the branches display a greater variety than in the case $d=2$, reflecting a more complicated energy landscape. Figure 8 shows the profiles of some critical potentials. Note that the potentials in dashed lines can be regarded as "anomalous" versions of their similarly extended counterparts, and have a lower energy. As can be expected, the potential branches which are maximizers for some $\gamma$ have a profile that is decreasing in $r$. 

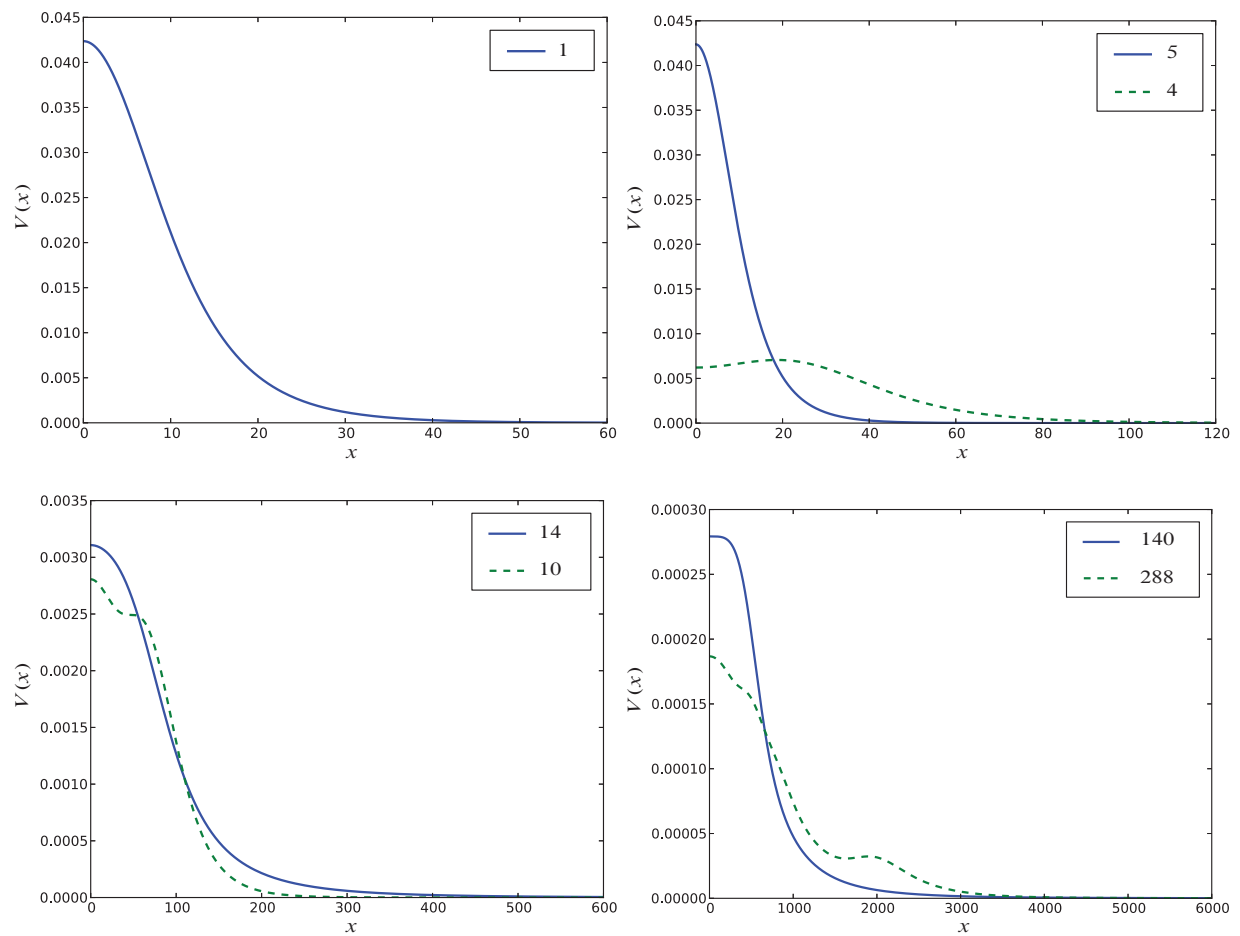

Figure 8. Shape of some critical points of $E(V)$, at $\gamma=1$. The qualitative shape of the curves does not change much when varying $\gamma$.

Table 3 displays the eigenvalue repartitions of a particular potential, the one with 140 bound states, at $\gamma=0.88$. Several patterns can be noted. First, for low values of $k$ and $l$, the approximate relationship $\lambda_{k+1, l}=\lambda_{k, l+2}$ holds. This is because the associated eigenfunctions are localized close to 0 . In this region, the potential can be approximated by a harmonic potential $V(0)+\frac{1}{2} V^{\prime \prime}(0) x^{2}$, which leads to the approximation

$$
\lambda_{k, l}=V(0)+\sqrt{V^{\prime \prime}(0)}\left(2 k+l+\frac{3}{2}\right),
$$

explaining the relationship $\lambda_{k+1, l} \approx \lambda_{k, l+2}$. This is only valid for small $k$ and $l$, where the harmonic approximation is valid. When the eigenvalues are close to zero, another pattern emerges: the last negative eigenvalues have the same value of $k+l$, leading to a triangular pattern in Table 3. This seems to be true for the maximizing branches $(1,5,14,140)$, but not for the others (which tend to have a different ordering of the eigenvalues close to zero). We do not have an explanation for this.

Every potential we were able to compute was below the $R=1$ threshold for $\gamma>1$. These results confirm the Lieb-Thirring conjecture that $\gamma_{c, 3}=1$. However, the subtle behavior near $\gamma=1$ means that there might exist maximizing potentials for $\gamma>1$ we were unable to find. 
Table 3. Eigenvalue repartition for the branch with 140 bound states, with $\gamma=0.88$, in units of $10^{-6}$ for readability. As in Section $2.2, \lambda_{k, l}$ is the $k$-th eigenvalue of equation (5). The positive eigenvalues are finite size effects and have no physical meaning, therefore we do not write them beyond the first one.

\begin{tabular}{|c|c|c|c|c|c|c|c|c|}
\hline$\lambda_{k, l}$ & $k=1$ & $k=2$ & $k=3$ & $k=4$ & $k=5$ & $k=6$ & $k=7$ & $k=8$ \\
\hline$l=0$ & -85 & -54 & -30 & -13 & -4 & -0.7 & -0.02 & +0.25 \\
\hline$l=1$ & -69 & -41 & -20 & -7.6 & -1.8 & -0.1 & +0.02 & \\
\hline$l=2$ & -54 & -29 & -12 & -3.4 & -0.3 & +0.03 & & \\
\hline$l=3$ & -39 & -18 & -5.8 & -0.7 & +0.04 & & & \\
\hline$l=5$ & -26 & -9 & -1.3 & +0.06 & & & & \\
\hline$l=6$ & -14 & -2.3 & +0.08 & & & & & \\
\hline$l=7$ & -3.8 & +0.1 & & & & & & \\
\hline
\end{tabular}

4.4. The case $\boldsymbol{d} \geq 4$. The results we obtained in the case $d \geq 4$ are similar to the case $d=3$, with $V_{\gamma, d, 1}$ as the maximizer for small $\gamma$, until it is outperformed by branches with a larger number of bound states, which all fall under the semiclassical limit before $\gamma=1$. However, the high cost of computation (the higher the dimension, the more ill-conditioned the eigenvalue problem is) prevents us from performing a systematic study.

\section{Conclusion}

In this paper, we used a maximization algorithm to investigate the best constants of the Lieb-Thirring inequalities, an important open problem of quantum mechanics. Discretizing the problem using finite elements, we were able to numerically compute critical points of the functional. Our main findings are listed below.

- In one dimension, the only critical point we found is the potential with exactly one bound-state $V_{\gamma, 1,1}$. For all initial data, the algorithm seems to either converge to this potential, or to split in separating bumps. This supports the conjecture that these potentials are the only maximizers of the functional for $\frac{1}{2} \leq \gamma<\frac{3}{2}$.

- In two dimensions, the only critical points we found were radial. For all initial data, the algorithm seems to either converge to a radial potential, or to split in diverging bumps. Among the radial potentials we were able to compute, $V_{\gamma, 2,1}$ was the maximizer for $\gamma<1.16$. After this point, the maximum corresponds to the semiclassical regime. The natural conjecture is that $V_{\gamma, d, 1}$ is the maximizer for $\gamma<1.16$, and therefore that $\gamma_{c, 2} \approx 1.16$.

- In three dimensions, branches of radial potentials with more than one bound state outperformed $V_{\gamma, d, 1}$. This confirms the theoretical result from [8], and provides new lower bounds (see Figure 7). The natural conjecture is that there is a non-decreasing sequence of integers $n_{\gamma}$ with $n_{\gamma} \rightarrow \infty$ as $\gamma \rightarrow 1$ such that the maximizer of the functional $\left(P_{\gamma, d}\right)$ has $n_{\gamma}$ bound states. 
- In dimension $d \geq 4$, the results are similar to the three-dimensional case, and we conjecture the same behavior.

Beyond these results, the study hinted at a very rich and highly nonlinear behavior of the maximizers of Lieb-Thirring inequalities. This study is based on a simple numerical method (finite element discretization). More involved computations could use a more appropriate Galerkin basis. This could allow for a more accurate computation of potentials with a larger number of bound states, and a more detailed exploration of the energy landscape.

On the theoretical side, the properties of the functional $\left(P_{\gamma, d}\right)$ remain unexplored. An open question is whether one can prove the existence of maximizers. The behavior of the maximization algorithm is also an interesting question, with the separation in bumps particularly interesting. Finally, we believe that our method could be adapted to generalizations such as models with positive temperatures or positive density [7], negative values of $\gamma$ (see [5]) or polyharmonic operators $(-\Delta)^{l}+V$ (see [22]).

\section{References}

[1] M. Aizenman and E. H. Lieb, On semiclassical bounds for eigenvalues of Schrödinger operators. Phys. Lett. A 66 (1978), 427-429. MR 0598768

[2] E. Cancès and C. Le Bris, On the convergence of SCF algorithms for the HartreeFock equations. M2AN Math. Model. Numer. Anal. 34 (2000), 749-774. MR 1784484 Zbl 1090.65548

[3] M. Cwikel, Weak type estimates for singular values and the number of bound states of Schrödinger operators. Ann. of Math. (2) 106 (1977), 93-100. MR 0473576 Zbl 0362.47006

[4] J. Dolbeault, Personal communication, 2012.

[5] J. Dolbeault, P. Felmer, M. Loss, and E. Paturel, Lieb-Thirring type inequalities and Gagliardo-Nirenberg inequalities for systems. J. Funct. Anal. 238 (2006), 193-220. MR 2253013 Zbl 1104.35021

[6] J. Dolbeault, A. Laptev, and M. Loss, Lieb-Thirring inequalities with improved constants. J. Eur. Math. Soc. (JEMS) 10 (2008), 1121-1126. MR 2443931 Zbl 1152.35451

[7] R. L. Frank, M. Lewin, E. H. Lieb, and R. Seiringer, A positive density analogue of the Lieb-Thirring inequality. Duke Math. J. 162 (2013), 435-495. MR 3024090 Zbl 1260.35088

[8] B. Helffer and D. Robert, Riesz means of bounded states and semi-classical limit connected with a Lieb-Thirring conjecture II. Ann. Inst. H. Poincaré Phys. Théor. 53 (1990), 139-147. MR 1079775 Zbl 0728.35078

[9] D. Hundertmark, E. H. Lieb, and L. E. Thomas, A sharp bound for an eigenvalue moment of the one-dimensional Schrodinger operator. Adv. Theor. Math. Phys. 2 (1998), 719-731. MR 1663336 Zbl 0929.34076

[10] E. Jones, T. Oliphant, P. Peterson et al., SciPy: Open source scientific tools for Python. http://www.scipy.org/ 
[11] A. Laptev and T. Weidl, Recent results on Lieb-Thirring inequalities. In Journées "Équations aux Dérivées Partielles". Held in La Chapelle sur Erdre, June 5-9, 2000. Université de Nantes, Nantes, 2000, Exp. No. XX. MR 1775696 Zbl 1135.81337

[12] A. Laptev and T. Weidl, Sharp Lieb-Thirring inequalities in high dimensions. Acta Math. 184 (2000), 87-111. MR 1756570 Zbl 1142.35531

[13] R. B. Lehoucq, D. C. Sorensen, and C. Yang, ARPACK users'guide: solution oflarge-scale eigenvalue problems with implicitly restarted Arnoldi methods. Society for Industrial and Applied Mathematics, Philadelphia, PA, 1998.

[14] A. Levitt, Convergence of gradient-based algorithms for the Hartree-Fock equations. ESAIM Math. Model. Numer. Anal. 46 (2012), 1321-1336. MR 2996329 Zbl 1269.82008

[15] E. H. Lieb, Bounds on the eigenvalues of the Laplace and Schrödinger operators. Bull. Amer. Math. Soc. 82 (1976), 751-753. MR 0407909 Zbl 0329.35018

[16] E. H. Lieb, Thomas-Fermi and related theories of atoms and molecules. Rev. Modern Phys. 53 (1981), 603-641. MR 0629207 Zbl 1049.81679

[17] E. H. Lieb, The stability of matter: from atoms to stars. Bull. Amer. Math. Soc. (N.S.) 22 (1990), 1-49. MR 1014510 Zbl 0698.35135

[18] E. H. Lieb and R. Seirnger, The stability of matter in quantum mechanics. Cambridge University Press, Cambridge, 2010. MR 2583992 Zbl 1179.81004

[19] E. H. Lieb and B. Simon, The Hartree-Fock theory for Coulomb systems. Comm. Math. Phys. 53 (1977), 185-194. MR 0452286

[20] E. H. Lieb and W. E. Thirring, Bound for the kinetic energy of fermions which proves the stability of matter. Phys. Rev. Lett. 35 (1975), 687-689.

[21] E. H. Lieb and W. E. Thirring, Inequalities for the moments of the eigenvalues of the Schrödinger Hamiltonian and their relation to Sobolev inequalities. In E. H. Lieb, B. Simon, and A. S. Wightman (eds.), Studies in mathematical physics. Essays in honor of Valentine Bargmann. Princeton Series in Physics. Princeton University Press, Princeton, NJ, 1976, 269-303. Zbl 0342.35044

[22] Y. Netrusov and T. Weidl, On Lieb-Thirring inequalities for higher order operators with critical and subcritical powers. Comm. Math. Phys. 182 (1996), 355-370. MR 1447297 Zbl 0865.47033

[23] C. C. J. Roothaan, New developments in molecular orbital theory. Rev. Mod. Phys. 23 (1951), 69-89. Zbl 0045.28502

[24] G. V. Rozenblum, Distribution of the discrete spectrum of singular differential operators. Dokl. Akad. Nauk SSSR 202 (1972), 1012-1015. English transl., Soviet Math. Doki. 202 (1972) 1012-1015. MR 0295148 Zbl 0249.35069

[25] M. Rumin, Balanced distribution-energy inequalities and related entropy bounds. Duke Math. J. 160 (2011), 567-597. MR 2852369 Zbl 1239.47019

[26] E. M. Stein and G. L. Weiss, Introduction to Fourier analysis on Euclidean spaces. Princeton Mathematical Series 32. Princeton University Press, Princeton, N.J., 1971. MR 0304972 Zbl 0232.42007

[27] T. Weidl, On the Lieb-Thirring constants $L_{\gamma, 1}$ for $\gamma \geq 1 / 2$. Comm. Math. Phys. 178 (1996), 135-146. MR 1387945 Zbl 0858.34075 
Received June 20, 2012

Antoine Levitt, Université Paris-Dauphine, CEREMADE, Place du Maréchal Lattre de Tassigny, 75775 Paris Cedex 16, France.

E-mail: levitt@ceremade.dauphine.fr 